\title{
A giant's shoulders: a Perspective from Professor Norman L. Allinger
}

\author{
Terry R. Stouch · Yvonne C. Martin
}

Received: 26 March 2011/Accepted: 4 April 2011/Published online: 13 April 2011

(C) Springer Science+Business Media B.V. 2011

If we can see further into the details of molecular structure, it is because we are standing on several strong sets of shoulders, of which Norman "Lou" Allinger's are one. Many of us remember the days when small molecule modeling was done almost solely with his "MM" series of programs, MM1, MM2, MM3, MM4 which Lou painstakingly parameterized for the particular chemical functionality of his research efforts. Far from the high dollar efforts and software industry we see today, Lou's software and molecular mechanics efforts never received funding; they were a labor of love piggy-backed on larger research efforts. He made source code available to anyone who wanted it and charged only a few thousand dollars to industrial users. Anyone interested in computational chemistry had-any many still have-a copy of "Burkert and Allinger" on their shelves. Now we are lucky that decades later Lou has sacrificed his retirement from The University of Georgia to provide us with a new edition. It is fair to say that without Lou's contributions the field of computational chemistry would be years behind where it is now.

In this issue Lou continues to teach and is good enough to provide his Perspective on today's role of molecular mechanics in this era where we almost blindly press a button to generate thousands of conformations of hundreds of thousands of molecules and simulate systems comprised of tens-to-hundreds of thousands of atoms. He reminds us of the hard work and detailed study that goes into getting

\footnotetext{
T. R. Stouch ( $\square)$

Science For Solutions, LLC, Princeton, NJ, USA

e-mail: tstouch@gmail.com

Y. C. Martin

Martin Consulting, Waukegan, IL, USA
}

the structure of just one bond or functional group correct and he allows us to infer the heartaches that can arise in this pursuit. He cautions us that quantum mechanics not only still does not fulfill our structural (not to mention temporal) needs, but also does not provide the chemical, structural, and physical insight that teaches us chemistry. We are implicitly reminded that as we push that button we should pay attention to the hard truth that there is still work to be done and there may lay inaccuracies underneath that button. In particular, Lou notes that the force fields so carefully crafted for the modeling of proteins are not crafted to meet the challenge of the greater chemical diversity of drugs or other molecules.

Lou takes us through the history and philosophy of the field and gives us specific examples of how molecular mechanics can help us understand structural chemistry. He refreshes us on the underpinnings of the science and mathematics of molecular mechanics. He illustrates the physical chemistry of molecular structure and provides examples of how physical phenomena affect 3D atomic structure. There is no one better than Lou to guide us in this study.

With their permission we provide part of an exchange between a knowledgeable reviewer and Professor Allinger which highlights some of the gems to be found in his Perspective:

Reviewer: The reviewer asks for some discussion of the force fields used in protein-ligand interaction studies. The implication from this manuscript is that these so-called protein force-fields do not adequately describe the structures of the ligands.

Allinger: The word "adequate" is key here. Protein force fields are generally what are referred to as Class 1 . That is, they are approximately harmonic and diagonal. 
Whether or not this is adequate depends on what it is that one is trying to calculate. An example is given in this article (Figures 3-6) where leaving out one of the crossterms in the force constant matrix, one gets a change in the potential energy surface of $4 \mathrm{kcal} / \mathrm{mol}$ ! In terms of an equilibrium constant, this would change an equilibrium from something like $97 / 3$ to $3 / 97$. I would assume that this would make quite a big difference.

Reviewer: Is the difference of $0.003 \AA$ in a bond length enough to influence interaction energy?

Allinger: The general answer would be in some cases small errors can lead to large consequences under some circumstances, as in the above example.

Reviewer: The MMFF force field has gained popularity-how does it stack up with respect to MM4?

Allinger: High accuracy (about one part per ten thousand) in molecular structures can be obtained only in microwave spectroscopy or by very accurate Schrödinger calculations for quite small molecules ( 3 or 4 atoms). Next best accuracy (approximately one part per thousand) can almost be obtained by MM4 (more like a few per parts per thousand here). It can also be obtained by some types of quantum calculations on sufficiently small systems, and it can be obtained in some cases experimentally by microwave or electron diffraction studies (again, on pretty small molecules). MMFF, in our hands, generally gave results with a general accuracy on the order of one part per hundred at best. That force field is basically Class 1, which causes part of the limitation. Further limitation comes from the fact that this force field is intended to be quite general, and many things that are not the same are taken to be the same (K. Kuchitsu and S. J. Cyvin in Molecular Structures and Vibrations, S. J. Cyvin, Ed., Elsevier Pub. Co., New York, 1972). This force field is designed to be applicable to just about everything, at the expense of the accuracy that might be obtained by more limited objectives, or by a great deal of additional work.
Reviewer: Should those interested in drug-biomolecule interactions use MM4 to study their small molecules? How might information gained be used?

Allinger: It would depend on what they were studying, how they were studying it, and what it was they were trying to learn. It would be of limited help to have an accurate MM4 structure for a small molecule, if this were going to be combined with much less accurate data from a large molecule, to give a combined result. Sometimes such a result would be useful. If the calculations are arranged so that the less accurate data cancel out in comparing two cases, for example, then the accuracy of MM4 calculation would be useful. But if that doesn't happen, it probably would not be very useful to make part of the calculation highly accurate, and then mix in some numbers that are much less so. (See back to the second paragraph of this response.)

Reviewer: There is a general statement that DFT doesn't work well.

Allinger: DFT is currently under active investigation by numerous research groups, and whether or not it works "well" is a relative term. The widely used B3LYP method does not usually work to "chemical accuracy."

Lastly, for those who haven't the pleasure to know Lou personally, he is a charming, understated, what used to be called a "gentleman scientist." As a graduate student one of us (TRS) personally benefited from the fact that he was always friendly and approachable and willing to talk science to anyone who was interested. Lou is also a Jazz pianist as depicted by the picture gracing the cover of this issue. Informal jam sessions by Lou and Dick Cramer on horn (featured in an earlier issue of this volume (J. CompAided Mol. Des. 2011, 25 (3))) created memorable moments at past Gordon Research Conferences. 